\title{
Towards a Taxonomy of KOS: Dimensions for Classifying Knowledge Organization Systems ${ }^{\dagger}$
}

\author{
Renato Rocha Souza*, Douglas Tudhope ${ }^{* *}$, and Maurício Barcellos Almeida**** \\ * Universidade Federal de Minas Gerais, Av. Borges de Medeiros, \\ 3501/504 Rio de Janeiro, RJ, Brazil, <rsouza@eci.ufmg.br> \\ $\because *$ Faculty of Advanced Technology, University of Glamorgan, Pontypridd, \\ CF37 1DL, Wales, United Kingdom, <dstudhope@glam.ac.uk> \\ ***: Escola de Ciência da Informação, Universidade Federal de Minas Gerais, \\ Av. Presidente Antônio Carlos, 6627, Campus Pampulha, 31.270-901, \\ Belo Horizonte, Minas Gerais, Brasil, <mba@eci.ufmg.br>
}

Renato Rocha Souza works as Researcher and Full Professor at Fundação Getúlio Vargas (FGV), Brazil and works as Colaborator Professor at Universidade Federal de Minas Gerais (UFMG), Brazil. He holds a Visiting Fellow at the University of Glamorgan, UK. He currently works with knowledge organization systems, natural language processing, and information retrieval systems.

Douglas Tudhope is Professor in the Faculty of Advanced Technology, University of Glamorgan and leads the Hypermedia Research Unit. His main current research interests are the intersecting areas of information science, digital libraries, and hypermedia and the semantic web. He has worked on various research projects applying knowledge organization systems to the cultural heritage domain, investigating topics such as semantic interoperability, terminology services, information extraction, and thesaurus-based query expansion. Since 1977, he has been Editor of the journal, New Review of Hypermedia and Multimedia. He serves as a reviewer for various journals and international programme committees.

Mauricio B. Almeida is currently an Associate Professor in the Department of Information Theory and Management at Federal University of Minas Gerais, Brazil. He collaborates with Ontology Research Group at University at Buffalo, US, working on medical vocabularies. Currently, his research involves ontologies and information systems.

Souza, Renato Rocha, Tudhope, Douglas, and Almeida, Maurício Barcellos. Towards a Taxonomy of KOS: Dimensions for Classifying Knowledge Organization Systems. Knowledge Organization. 39(3), 179-192. 27 references.

\begin{abstract}
This paper analyzes previous work on the classification of Knowledge Organization Systems (KOS), discusses strengths and weaknesses, and proposes a new and integrative framework. It argues that current analyses of the KOS tend to be idiosyncratic and incomplete, relying on a limited number of dimensions of analysis. The paper discusses why and how KOS should be classified on a new basis. Based on the available literature and previous work, the authors propose a wider set of dimensions for the analysis of KOS. These are represented in a taxonomy of KOS. Issues arising are discussed.
\end{abstract}

Received 7 September 2011; Revised 1 February 2012; Accepted 23 February 2012

$\dagger$ This is an extended and revised version of a paper presented at the 2010 ISKO Conference in Rome.
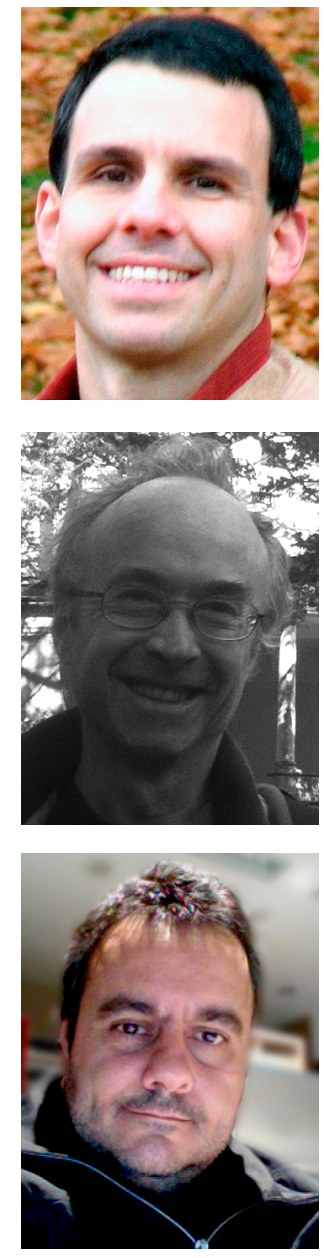

\footnotetext{
This is an extended and revised version of a paper presented at the 2010 ISKO Conference in Rome.
} 


\subsection{Representation and Knowledge Organization Systems}

Although central to many scientific fields, or perhaps because of that, the process of representing knowledge is not a simple matter. There are many distinct theories, models, methodologies, and products; all influenced by specific applications, backgrounds, and purposes. Knowledge representation artifacts are produced in related fields, such as artificial intelligence, semiotics, computer science, and cognitive science, but are also widely used in a myriad of less related areas, such as education, mathematics, business modeling, linguistics, and many more.

The field of library and information science (LIS), however, considers these representational artifacts as a paramount matter. In fact, LIS takes upon itself the task of organizing and facilitating the retrieval of the wealth of information that arises from the knowledge produced in all other fields, and this involves the creation of epistemological and ontological surrogates. Many of the LIS theories, processes, and instruments are dependent on representation products, modeled through successive abstractions over the relevant characteristics of a chosen world or domain, or the information gathered and processed about these, registered in information systems and documents. Those representations, known as knowledge organization systems (KOS), vary enormously in format and display, but they share the general characteristic of aiding knowledge elicitation and organization, aiming at promoting the retrievability of information.

KOS are not new to librarians or biologists, who have been using them over centuries for catalogs, bibliographic classification systems, and taxonomies. However, they have received special attention nowadays in contexts like the Semantic Web, given the need for vocabulary disambiguation and the highly formalized structures needed to allow machine "semantics" and "understanding." The W3C has encouraged the publication of KOS in the Semantic Web for aiding semantic interoperability, information retrieval, and access to information resources (http://www.w3.org/ 2001/sw/wiki/SKOS/FAQs). Besides the general web oriented languages and schemes for knowledge representation, such as RDF and OWL, there are standards, such as SKOS (W3C 2009), aiming to bridge the gap between LIS KOS and logic based formal structures, by expressing thesauri and related schemes in the Semantic Web environment; or the new microformats standards that have been proposed by Google and other players for better searching experience.
Knowledge of the KOS and its characteristics is necessary for the responsible design of any information retrieval system or knowledge base system, especially in the digital environment, allowing the architects to make grounded decisions on the project.

Taking a broad view, there are hundreds of types of KOS. From thesauri and ontologies to the inverted indexes of information retrieval systems (IRS), from the surrogate files to the documents' abstracts, KOS are hard to compare within a single scope or framework. Nevertheless, many attempts have been presented, although seldom addressing the subject holistically. The goal of this paper is to analyze some of the previous KOS classification attempts, discussing strengths and weaknesses, and to propose a new and integrative framework. The paper discusses why and how the KOS should be tentatively classified on a new basis. Based on the available literature and previous work, the authors propose a wider set of classification dimensions expressed as a taxonomy of KOS.

\subsection{A typology of KOS}

Perhaps the first question we should try to answer is: what is a KOS? No matter how extensive, all lists attempting to enumerate all possible KOS will fail under other eyes' perspective, as long as the different interpretations about what may be called a KOS lead to different results. Many candidate lists have been attempted (Hodge 2000; Bergman 2007; Tudhope et al. 2006; Wright 2005, 2008; BSI 2007). According to Hodge (2000), KOS are at the heart of the library environment. She defines a broad view of KOS as something that:

encompass all types of schemes for organizing information and promoting knowledge management. Knowledge organization systems include classification and categorization schemes that organize materials at a general level, subject headings that provide more detailed access, and authority files that control variant versions of key information such as geographic names and personal names. Knowledge organization systems also include highly structured vocabularies, such as thesauri, and less traditional schemes, such as semantic networks and ontologies.

Wright $(2005,2008)$ is concerned to encompass wider contexts of use than the LIS focus ultimately on retrieval purposes, and, in particular, she is concerned with "language purposed" applications (translation 
tools, etc.) She considers KOS as one kind of KRRs (Knowledge Representation Resources) and alternatively names KOS as "Knowledge Organization Schemes." Importantly, she identifies communities of practice as an important organizing principle; different communities define KOS differently, according to their practical purposes.

In fact, the "terminology relating to terminology" is often confusing (Hodge 2000), as is the "concept of concept" (Klein and Smith 2005), which renders more difficult the task of knowledge representation. Regarding this issue, we have adopted a comprehensive and lato sensu approach, considering as KOS "all types of schemes for organizing information and promoting knowledge management" (Hodge 2000), and building on previously cited works. In this sense, we consider KOS as knowledge representations based on concepts and with different degrees of relationships among them. Figure 1 presents a summary of the KOS collected by the authors, as a concept mapitself a type of KOS.

As seen in the map, the first criterion for division was the KOS structure type, with a secondary division taking account of different application domains and use cases. The main types range from Unstructured Texts to those that regard Concepts, Relationship and Layout as part of the structure. The ones classified under Term and/or Concept Lists present simple structures (mainly alphabetical displays, but usually no hierarchies), and the Concept and Relationship Structures comprises a large range of structures that present some different degree of relationship expressiveness. The simpler ones present hierarchies with loose hyponym/hyperonym relationships, but Thesauri, for instance, may include meronomy along with some nonspecified associative relationship; and Formal Ontologies allow the representation of all sorts of relationship types, depending on the expressiveness of the language used for representing them. It is evident, at this point, that one cannot escape arbitrariness when trying to classify things in general, and this KOS classification is not an exception. Hence, it is useful to comment on the main differences from the previous KOS taxonomies proposed by Hodge (2000) and Wright (2008) and this concept map:

Firstly, in spite of the heterogeneity, we decided to be inclusive in our approach. Therefore, Abstracts, Concordance Lines and IR indexes, among others,

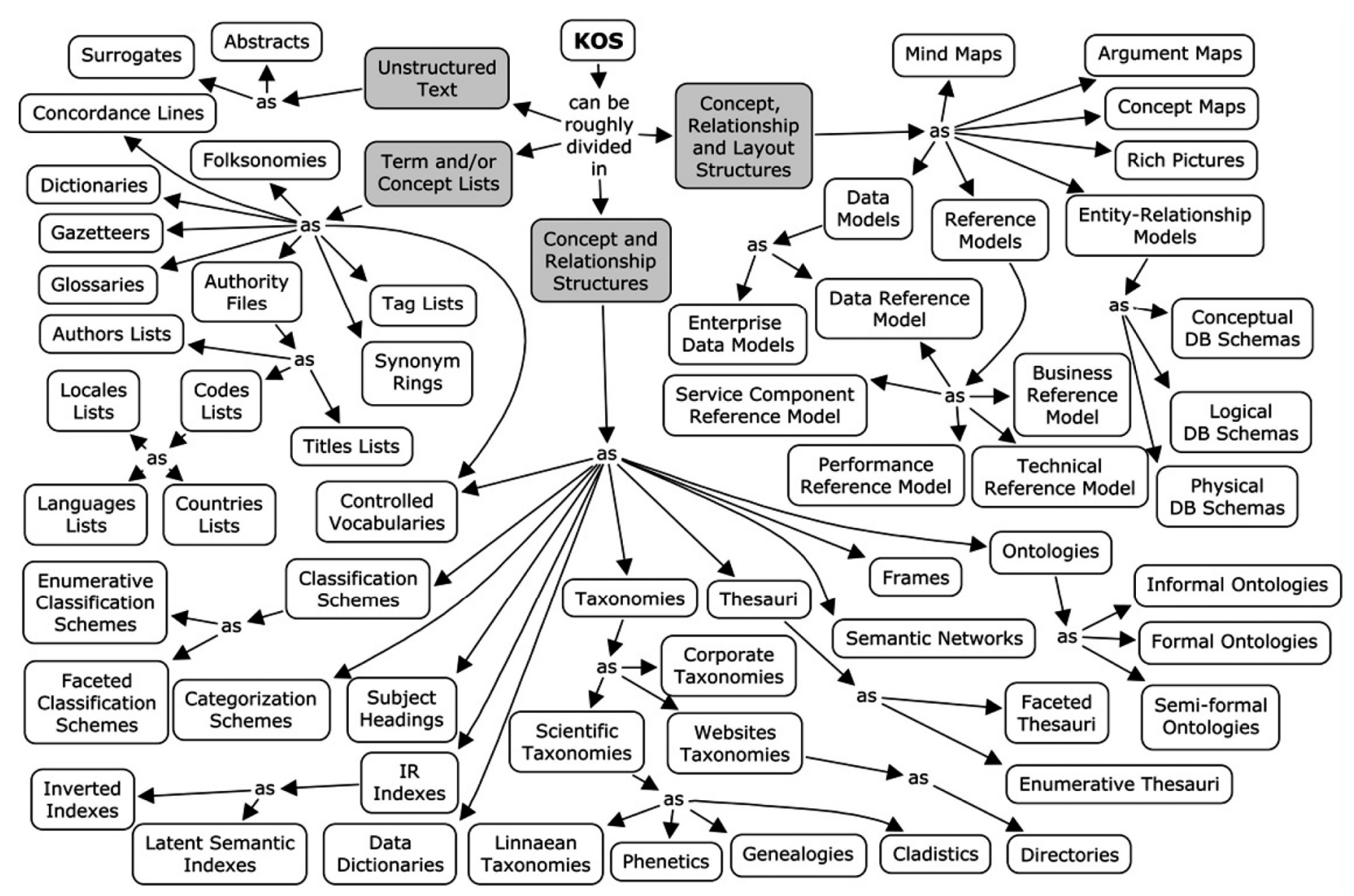

Figure 1. A tentative set of types of KOS 


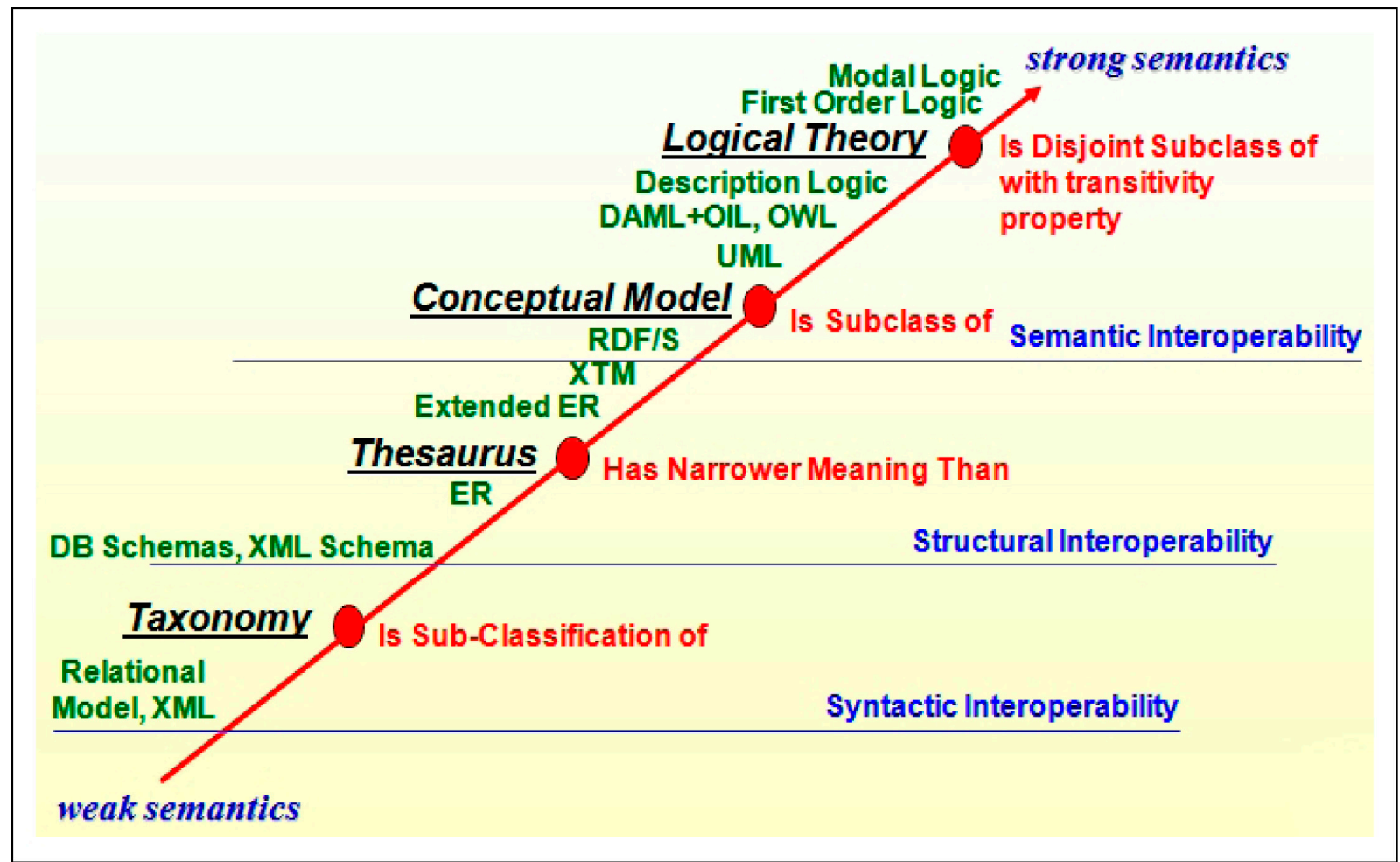

Figure 2. KOS Spectrum from Obrst (2004)

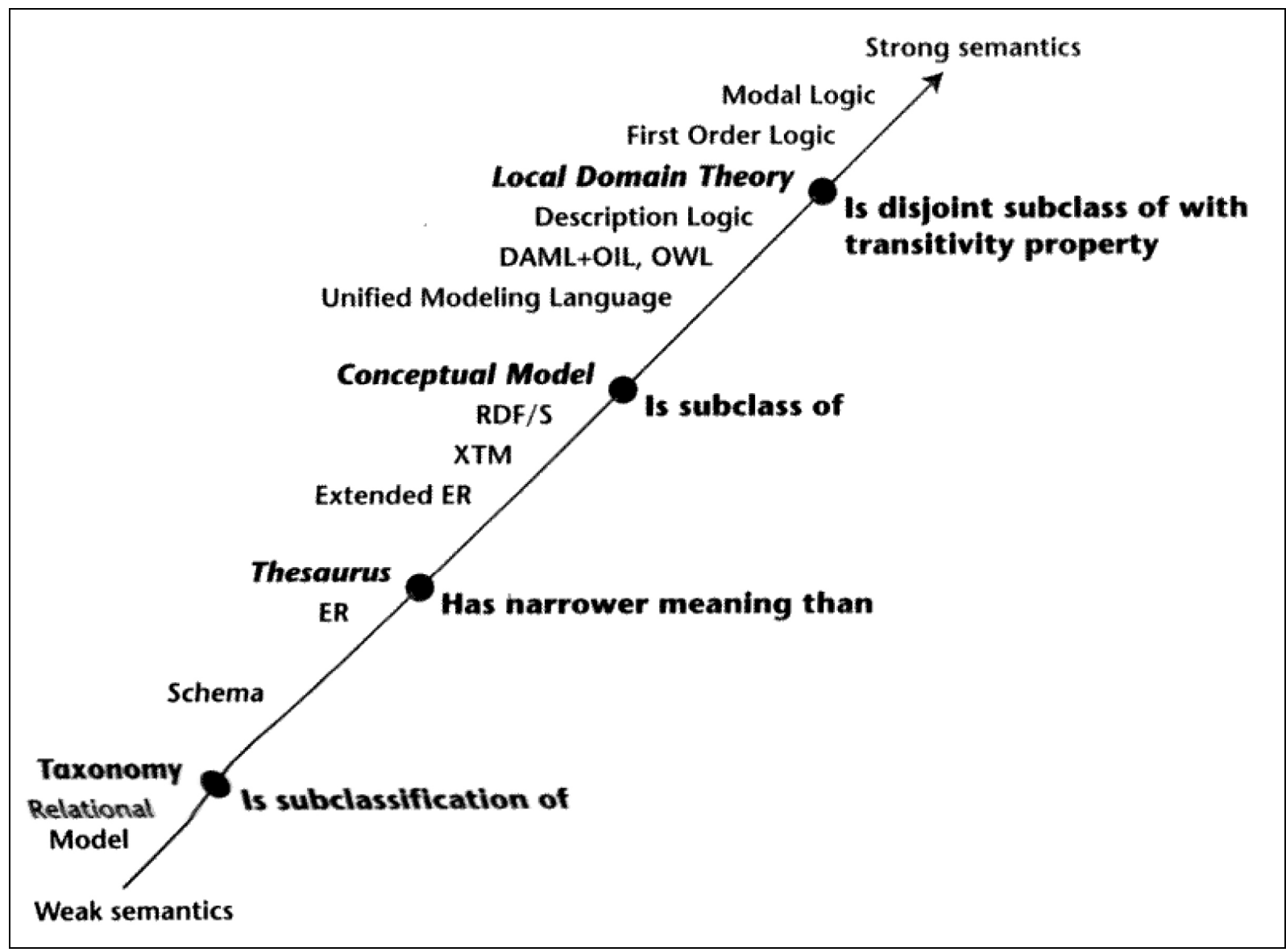

Figure 3. KOS Spectrum from Daconta et al. (2005) 
were included as KOS because: i) they are used for knowledge organization and information retrieval; ii) they promote knowledge management; iii) they are knowledge representation structures based on terminology. Using the same rationale, we have excluded the standard formats (as HTML, SGML, etc.) and products such as WordNet, as specified by Wright (2008), because the former are tools to represent $\mathrm{KOS}$, and the latter is an instance of a semantic network/lexical database.

Lastly, it is important to highlight that many structures (like controlled vocabularies) could have been classified otherwise, according to the sense chosen and example taken. Furthermore, the representation in Figure 1 does not show the breakdown of all concepts, such as semantic networks, due to the space limits of the concept map, but that could be easily achieved. This map was presented solely for the purpose of revealing the complexity of different kinds of KOS, and, as we are arguing, no single classification approach would be appropriate or exhaustive.

It is expected that many other structures can be suggested as inclusions; like most typologies, this one will always be a work in progress.

\subsection{Review of KOS spectra}

Despite its importance, there is little agreement on how to classify KOS and what might be the parameters or dimensions with which they could be classified. Traditionally, the different types of KOS tended to be used inside particular communities of practice, with little need for comparison and classification of the various types. In recent years, with the growth of networked computer based applications and wider use and cross use of KOS, this has changed to some degree. This partly results from the need to map (or cross walk) between different types of KOS and partly reflects the wider choice of online KOS available to a vocabulary developer or software application developer. Some reviews of types of KOS have taken place within the NKOS community (NKOS: Networked Knowledge Organization Systems/Services-http:// nkos.slis.kent.edu/), for example Hodge (2000) and Tudhope et al. (2006).

Other work has placed the different types of KOS in a form of classification spectrum, and some representative examples are reviewed in this section. Most of this work has probably taken place within the context of the Semantic Web and tends to reflect its origins in the artificial intelligence tradition and the use of formal ontologies to model the entities in a par- ticular world. Here the main concern tends to be suitability for logic-based automatic inferencing.

We should bear in mind that some of the following examples were partly illustrative in intent, and some of the sources acknowledge that they are intended to show a particular viewpoint. However, in our view, they reflect a fairly common characterization, which has led to confusion about the use and comparison of KOS more broadly. The consequence is comparisons of different types of KOS based upon a single dimension of analysis.

For example, "semantic strength" is employed by Obrst (2004) and Daconta et al. (2005), as in Figure 2 and Figure 3. They are generally concerned to outline ecommerce possibilities for data integration and interoperability associated with the Semantic Web, where ontologies can represent the semantics of key enterprise entities. The "strength" of the semantic, in these cases, is linked to "semantic expressivity," associated with the tractability of the KOS for different kinds of formalism. We can observe that these presentations tend to treat KOS and their representational languages alike.

Building on this work, Bergman (2007) adds "Time/Money" as a comparison characteristic, in addition to semantic strength, asserting that the more semantically strong (the more formal they are), the more expensive they are to build. Even if we were to grant that this trade off might hold, there is still an assumption of a single, shared purpose. Furthermore, the rationale for the relative positioning of the KOS remains unclear (Figure 4).

Another approach to represent different kinds of KOS is the spectrum proposed by Almeida et al. (2011), which makes a separation between use by humans and use by machines (Figure 5). It is still essentially a one dimensional spectrum, based on the formality of the semantics. However, it adds an additional element of a functional characteristic, represented by icons associated with each element: instrument for information organization, data model for proceduraloriented systems, Web-based declarative language, data model for web-based declarative-oriented system, webbased logic language, logic language. These serve to identify clusters, within which comparison of the underlying semantics is more meaningful.

The preparation for a discussion panel on ontologies at the 1999 American Association for Artificial Intelligence National Conference (AAAI-99 with panelists: Uschold, Gruninger, Lehmann, McGuinness) gave rise to another spectrum (Figure 6) on different possible definitions of ontology, based on an "axis of axiomatization," a key distinction being the 


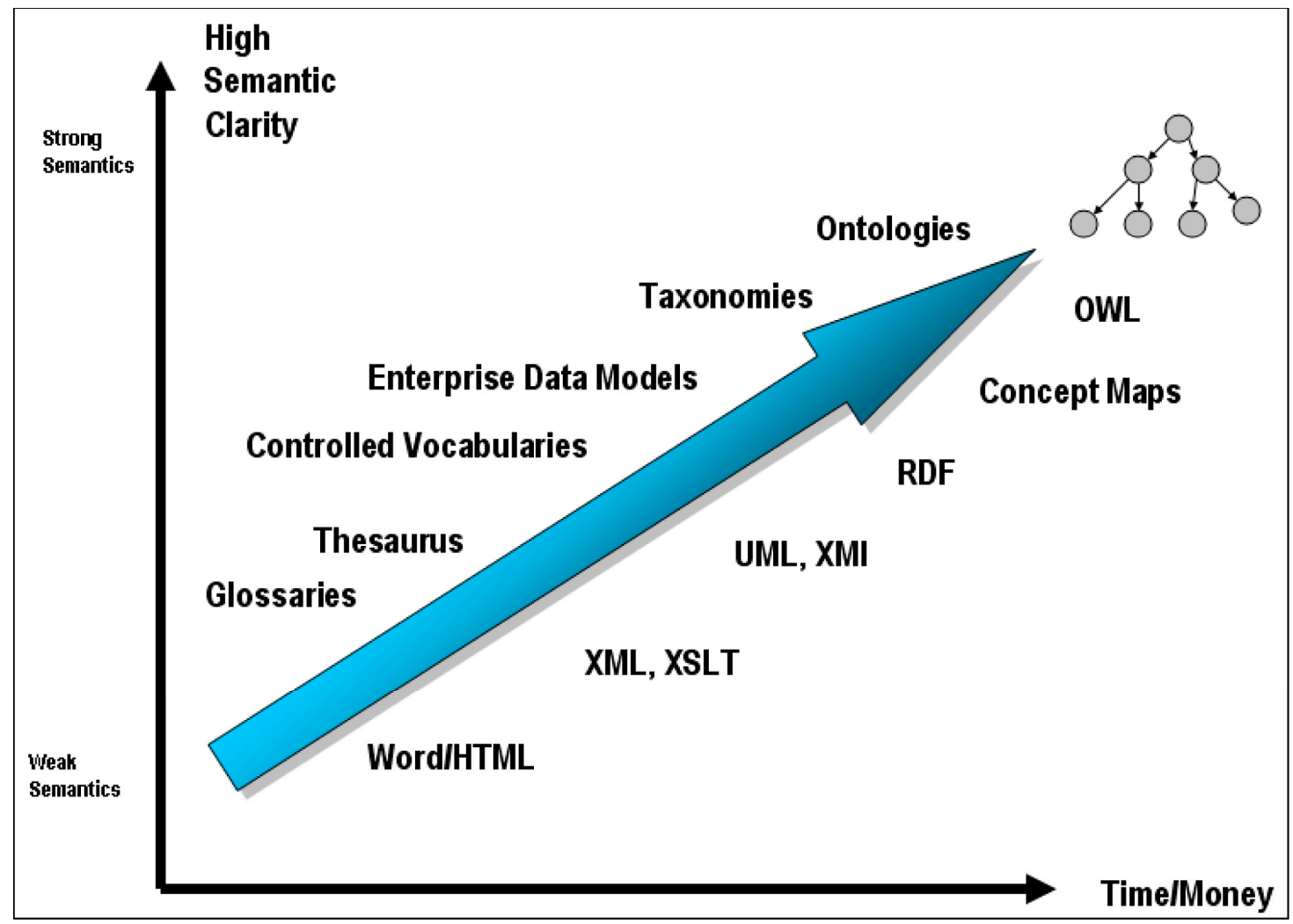

Figure 4. KOS Spectrum from Bergman (2007)

possibilities for automated (logical) reasoning. Although this is presented as a single characteristic, as we shall argue later, various factors are involved.

This spectrum has been influential for subsequent characterisations. McGuiness refined the 1999 panel model in two further papers (Lassila and McGuinness 2001; McGuinness 2003) offering the same general argument, along with a more detailed presentation of semantic relationships (see the spectrum in Figure 7). Taxonomies are included in the spectrum in Smith and Welty (2001), but omitted in Welty (1999) and by McGuinness. The single dimension in Figure 7 is similar to "semantic strength," although here this is illustrated and further defined via different types of semantic relationship. The single purpose is suitability for reasoning with formal logic, based on the formality of relationships, together with provision of instances, properties, constraints, as can be seen from the 1999 AAAI Panel original version. All types of KOS are represented as ontologies (Figure 7).

Guarino (2006) uses the term "ontological precision" in a similar way (Figure 8), though "ontological" refers in this case to the exactness with which the referred domain can be represented. Note that taxonomies are included again in this spectrum, albeit at a different position from Bergman (2007) and Smith and Welty (2001). Ontologies can be classified "according to their accuracy in characterizing the conceptualization they commit to" (Guarino 1998). He goes on to say that this can be achieved by a richer axiomatization or by a richer set of conceptual relations. While beyond the scope of this paper, we can note that Guarino distinguishes between different types of ontologies according to level of generality or dependence on a particular point of view, for example formal top-level ontologies versus domain versus application ontologies. The upper ontologies have wider potential uses, while lower ontologies may be subsequent specializations. It can be seen that the accuracy or precision of domain representation is again for purposes of logical computer reasoning.

Zeng (2008), building on and extending the spectrum in (Zeng and Salaba 2005) and NISO (2005), makes a useful contribution by expanding the $\mathrm{X}$-axis to show the functions associated with the different KOS and their semantic relationships (Figure 9).

This allows for more specific comparisons. The ordering of the functions gives the spectrum a similar shape to the spectra previously described, which emphasize purposes of logical reasoning. There are still, however, inevitable simplifications arising from the presentation as an essentially one-dimensional spectrum. 


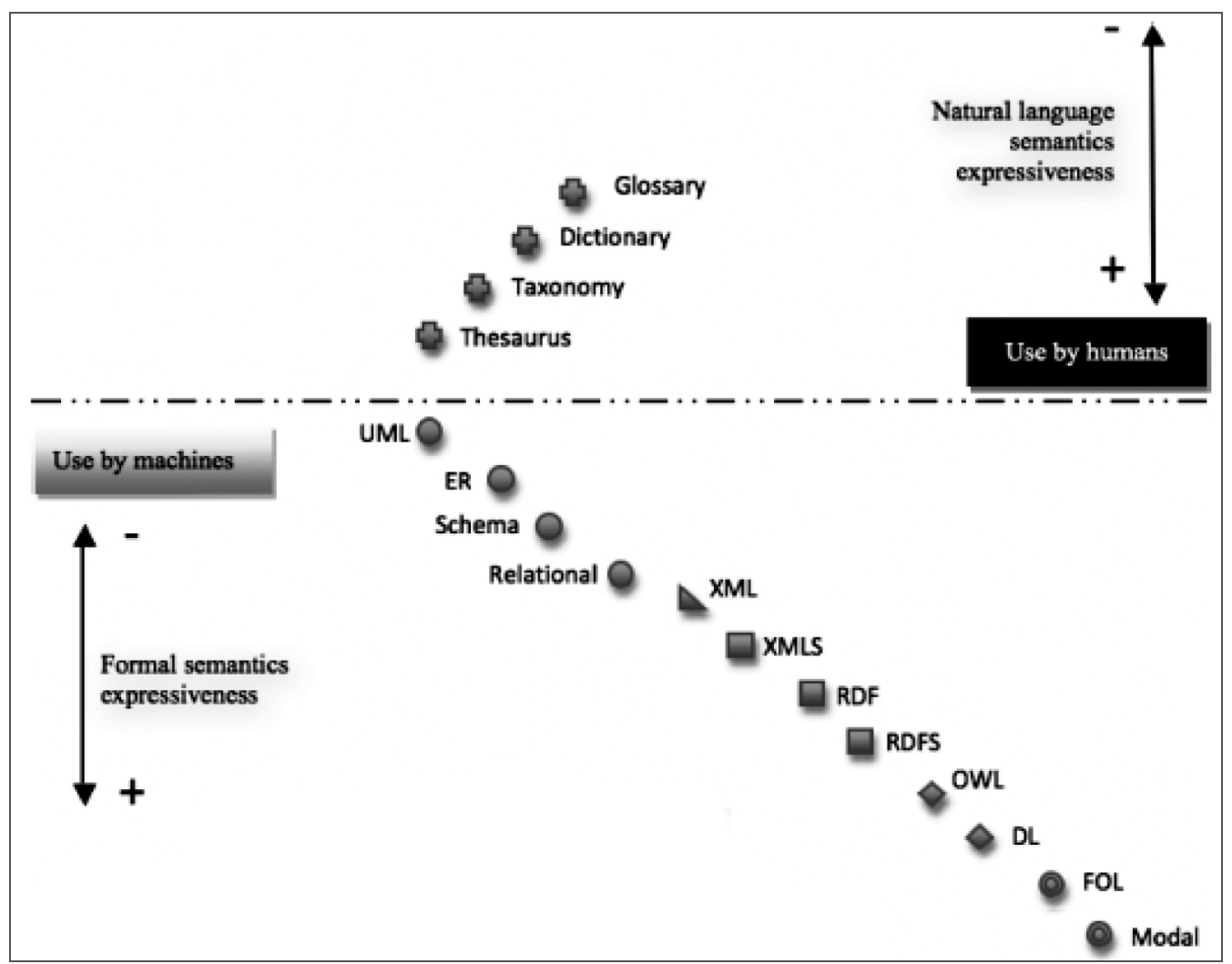

Figure 5. Spectrum proposed by Almeida, Souza and Fonseca (2011)

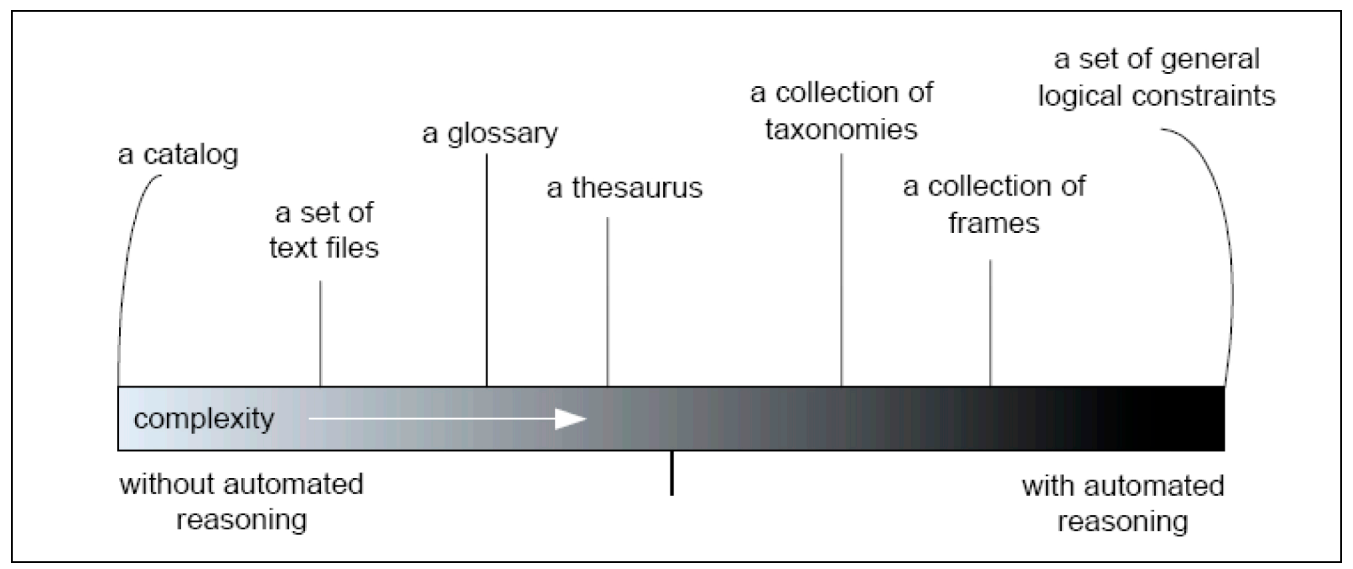

Figure 6. KOS Spectrum slightly adapted from Smith \& Welty (2001) based on Welty (1999)

While the description of functions may hold in the general "ideal type" of KOS, particular instances of a KOS type may differ in important characteristics. For example, some particular classification schemes may have more complex semantic relationships and dimensions of structure than most thesauri, which might be considered to outweigh (in terms of semantic complexity) the provision of associative relationships. Many formal ontologies, on the other hand, lack systematic provision for synonym control, and, indeed for some ontologies, that is not part of the intended purpose.

\subsection{Deconstructing the spectra}

Various issues arise from the review of KOS spectra in the previous section. These spectra tend to focus on a single element as the basis for comparison, leaving many key issues unexplored, while some only consider subsets of the structures accepted as KOS. They thus tend to be presented as one-dimensional spectrum, or two (we would argue correlated) dimensions. The visual rhetoric is that of a linear progression, with ontologies as the ultimate form. 


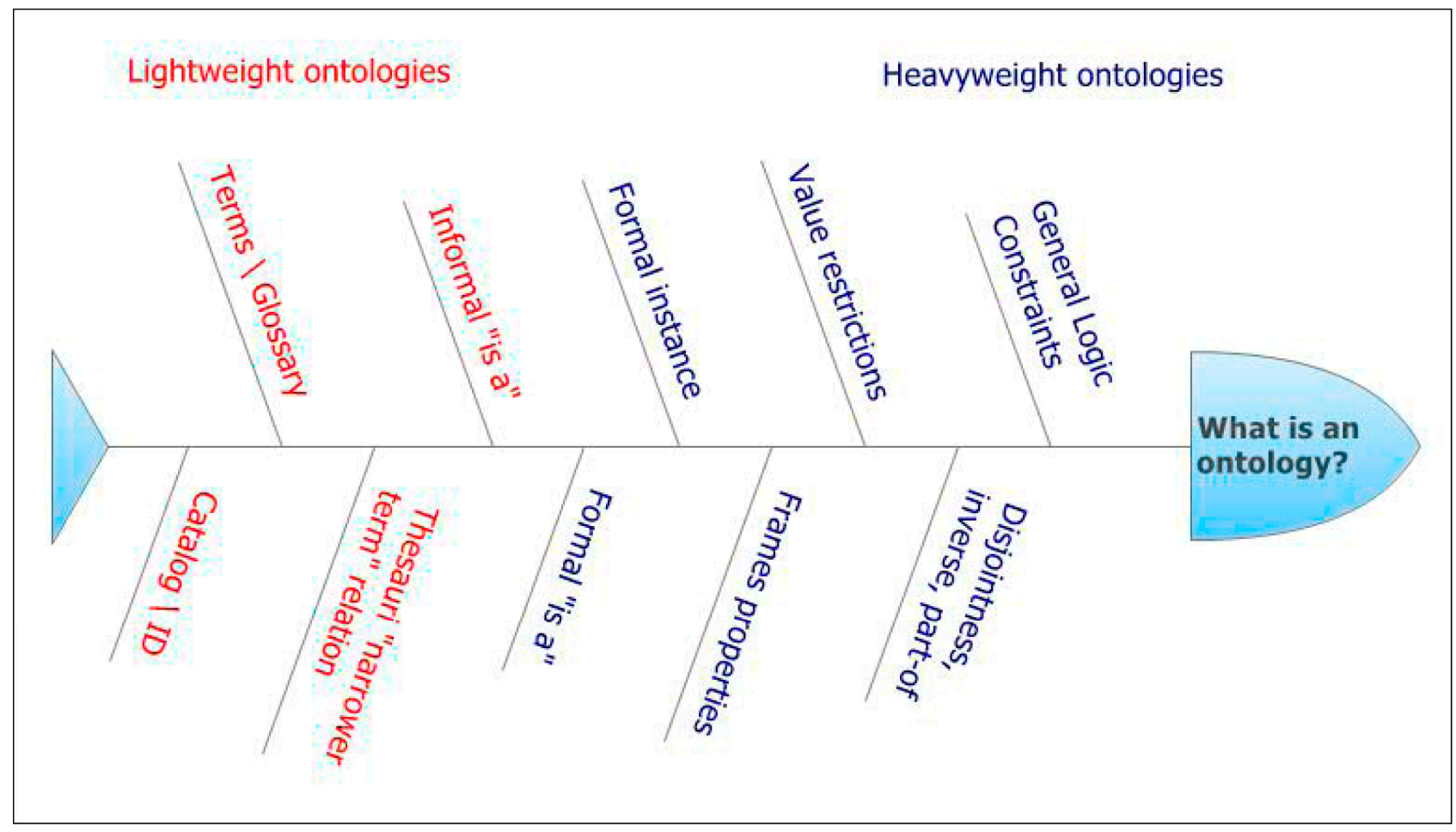

Figure 7. KOS Spectrum (adapted from presentation) from Lassila \& McGuinness (2001)

\section{Levels of Ontological Precision}

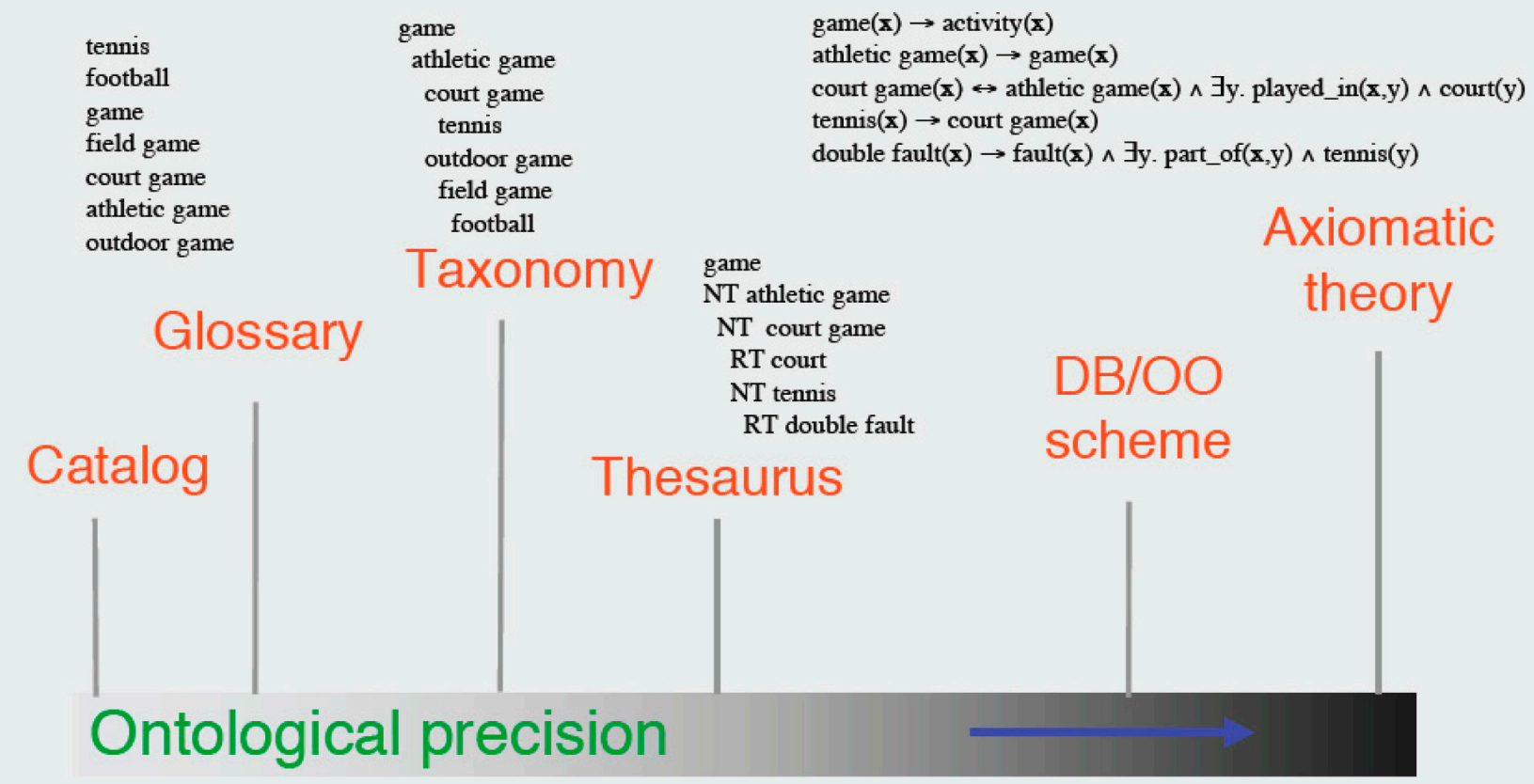

Figure 8. KOS Spectrum from Guarino (2006) 


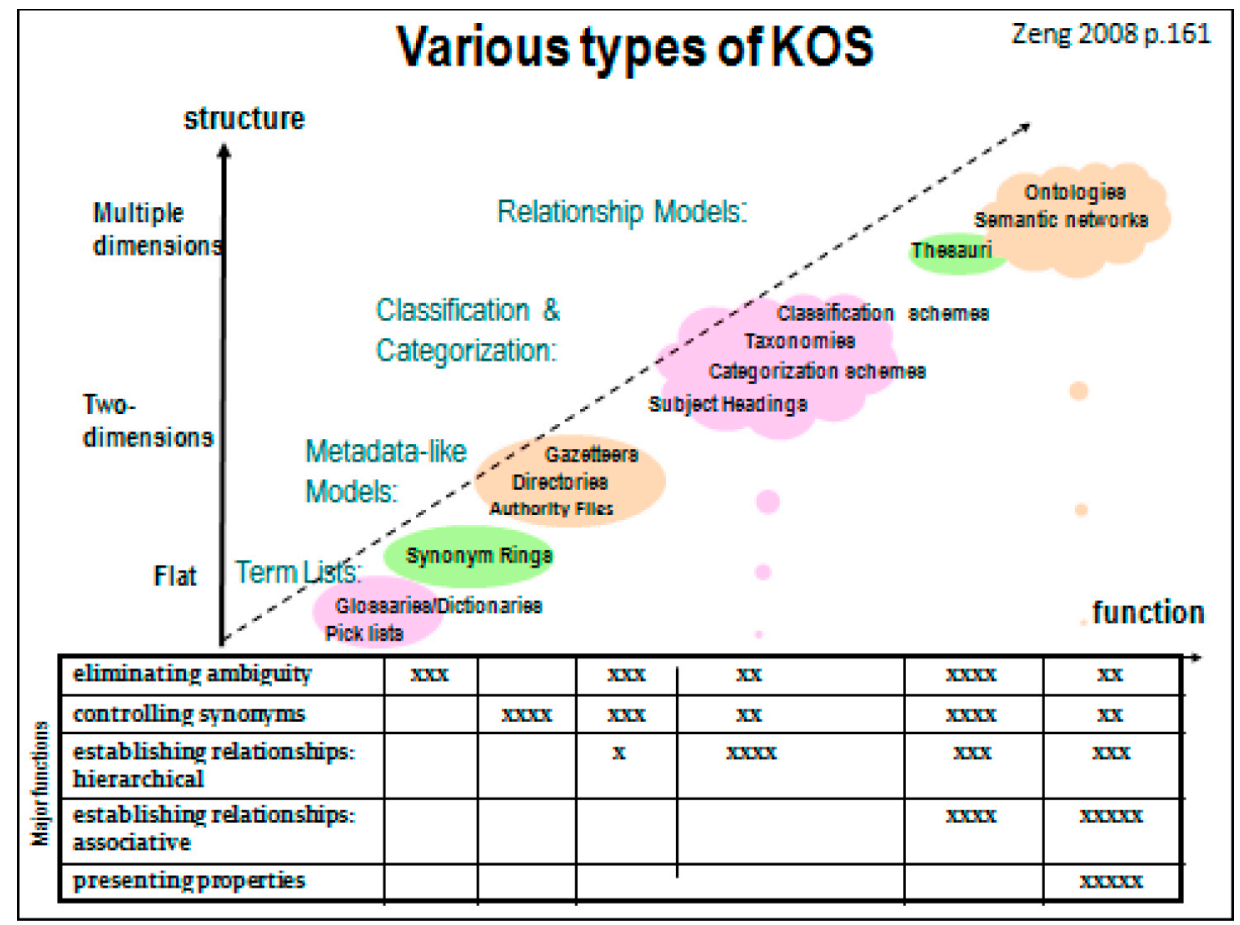

Figure 9. KOS Spectrum from Zeng (2008)

There is confusion as to the precise meaning of the terminology for different types of KOS in the spectra reviewed. The range of interpretations for the term taxonomy, for example, varies considerably. We can see how different authors tend to think differently, asserting that taxonomies are less (Obrst 2004; Daconta et al. 2005; Guarino 2006; Zeng and Salaba 2005) or more (Bergman 2007; Smith and Welty 2001) structured than thesauri. This illustrates the lack of agreement about what constitutes a taxonomy and the lack of terminology control in work on controlled terminologies. While precise definitions are beyond the scope of this paper, we generally follow BSI 8723 (2007) (a more extensive set of definitions will result from the ISO 25964 activity, supporting the development and application of thesauri in the context of networking opportunities; part 1 will focus on thesauri for information retrieval and Part 2 on interoperability with other vocabularies: http://www.niso.org/workrooms/ iso25964) and the new ISO 25964 Thesaurus Standard (Dextre Clarke 2012).

The meaning of ontology also varies considerably. Following a common practice in some Semantic Web literature, McGuinness (2003) employs the term for all vocabularies associated with Semantic Web applications (see Figure 7). While that paper does outline some uses of "lightweight" ontologies, such as navigation support, browsing, search, and query expansion, in our view, the blurring of distinctions entailed in the loose use of the term is unhelpful. It tends to obscure the details of differences between KOS and the specific purposes each has been designed for. It might be asserted that all KOS are the products of some kind of ontological modeling, but using the term "ontologies" arbitrarily can cause confusion.

The scope of the comparison also tends to vary. There can be wide variation in the particular instances of an ideal KOS type which may differ in important characteristics. In some cases, KOS designed for very different purposes are compared, where the scope and extent of their application may be very different.

For example, in some of the KOS spectra presentations, it can be noticed that there is no distinction between the types of KOS and the languages that can be used for representing them. Also, no distinction tends to be made between the ideal type of a KOS and specific instances of that type. This poses problems for the many hybrid "chimerical" creatures within the menagerie of KOS that have been developed or proposed. Some comparisons are only meaningful if applied to KOS instances, and some are related to the decisions on the implementation, since the same system can be represented in different ways. For detailed KOS classification, more aspects and dimensions are required than can be presented in a one or two dimensional spectra.

Some previous work has attempted comprehensive KOS comparison dimensions without producing 
spectra (including Soergel 2001a and 2001b; Tudhope 2006; Wright 2005, 2008). There is some commonality and also difference, according to the characteristics taken into consideration. In this regard, we have compared, adapted and sometimes discarded the characteristics reviewed, in order to compose the dimensions proposed in this paper. Table 1 shows the set of characteristics taken into account from the various sources considered in this paper.

\begin{tabular}{|c|c|}
\hline $\begin{array}{l}\text { Almeida, Souza } \\
\text { and Fonseca, } 2011\end{array}$ & $\begin{array}{l}\text { Representational power, Seman- } \\
\text { tic Expressiveness, Intelligibility } \\
\text { (for Humans), Formalization } \\
\text { (machine oriented) }\end{array}$ \\
\hline Bergman, 2007 & Semantic Strength, Time/Money \\
\hline Guarino, 2006 & Ontological Precision \\
\hline Hodge, 2000 & $\begin{array}{l}\text { Structure and complexity, Rela- } \\
\text { tionship between terms, Histori- } \\
\text { cal function }\end{array}$ \\
\hline $\begin{array}{l}\text { Lassila \& } \\
\text { McGuinness, } \\
2001\end{array}$ & $\begin{array}{l}\text { Ontology Level (formality of } \\
\text { semantic relationships), logical } \\
\text { reasoning }\end{array}$ \\
\hline $\begin{array}{l}\text { Obrst, 2004; } \\
\text { Daconta et al, } \\
2005\end{array}$ & Semantic Strength \\
\hline $\begin{array}{l}\text { Smith \& Welty, } \\
2001\end{array}$ & Complexity, logical reasoning \\
\hline $\begin{array}{l}\text { Soergel, 2001a } \\
\text { and 2001b }\end{array}$ & $\begin{array}{l}\text { Purpose, Coverage of concepts } \\
\text { and terms, Sources, Quality of } \\
\text { usage analysis, Conceptual analy- } \\
\text { sis and conceptual structure, } \\
\text { Terminological analysis, Use of } \\
\text { precombination in the index lan- } \\
\text { guage, Access and display, For- } \\
\text { mat of presentation of the vo- } \\
\text { cabulary, Updating }\end{array}$ \\
\hline Tudhope, 2004 & $\begin{array}{l}\text { Entities (types, coordination, si- } \\
\text { ze, depth), Relationships (types, } \\
\text { expressiveness, formality), Typi- } \\
\text { cal application to objects in do- } \\
\text { main of interest (purpose), Rela- } \\
\text { tionship applying concepts to ob- } \\
\text { jects in domain }\end{array}$ \\
\hline $\begin{array}{l}\text { Wright, } 2006 \\
\text { and } 2008\end{array}$ & $\begin{array}{l}\text { Communities of Practice, Sys- } \\
\text { tematic resources, Non- } \\
\text { systematic resources, Technology } \\
\text { orientation, Degrees of indeter- } \\
\text { minacy, Language \& knowledge- } \\
\text { oriented standards, Standards } \\
\text { bodies }\end{array}$ \\
\hline Zeng 2008 & $\begin{array}{l}\text { Structure, semantic relation- } \\
\text { ships/functions }\end{array}$ \\
\hline
\end{tabular}

Table 1. KOS Dimensions proposed in the literature.

The descriptions that did not aim at producing spectra (Soergel, Tudhope, and Wright) have included various specific attributes, extending from the nature of the KOS entities and relationships to the context of the resulting application. All these descriptions mention the possibility of a faceted treatment of KOS description (which is beyond the scope of this paper but an interesting possibility for future work). All also include purpose or community of practice as an important element for consideration.

\subsection{A possible taxonomy of KOS dimensions}

In the model presented here, we have tried to capture the KOS characteristics discussed above in a coherent and integrated way, dealing with some slight differences in the meaning of the characteristics, without omitting any important dimension. We have added explicitly some dimensions that were only implicitly stated, or have never been covered before, such as media and sensitivity. We have also proposed a scale of values for some of the dimensions, in order to allow comparison and classification of KOS over certain predefined parameters.

The typology model is presented as a taxonomy, showing the different dimensions of the model, in a basic hierarchical structure (Figure 10). We chose to represent the typology in this manner rather than the linear presentation of the KOS spectra reviewed in section 3, in order to emphasize the complexity of KOS characteristics and to make the case that a one dimensional display does not capture the richness of the issues involved.

In the taxonomy, KOS characteristics are divided at the top level into intrinsic and extrinsic, and the former are subsequently divided into essential and accidental. The intrinsic dimensions are related to the KOS type per se, taken as an ideal entity, isolated from any users and the environment. Among the intrinsic, the essential are closely related to the type (or the broad "class" to which the KOS belong), independent of any particular application or implementation. The accidental, by their turn, encompass characteristics of a given instance of the KOS, and can be different for each implementation of the same intellectual work, with different levels of information carried. The extrinsic dimensions are related to the environment in which the KOS is used. The dimensions can be examined in Figure 11 and are discussed further in the following paragraphs.

The intrinsic/essential are divided into structural characteristics and standardization. The structural comprises characteristics of the entities and relationships that are presented in the KOS. For the entities, 


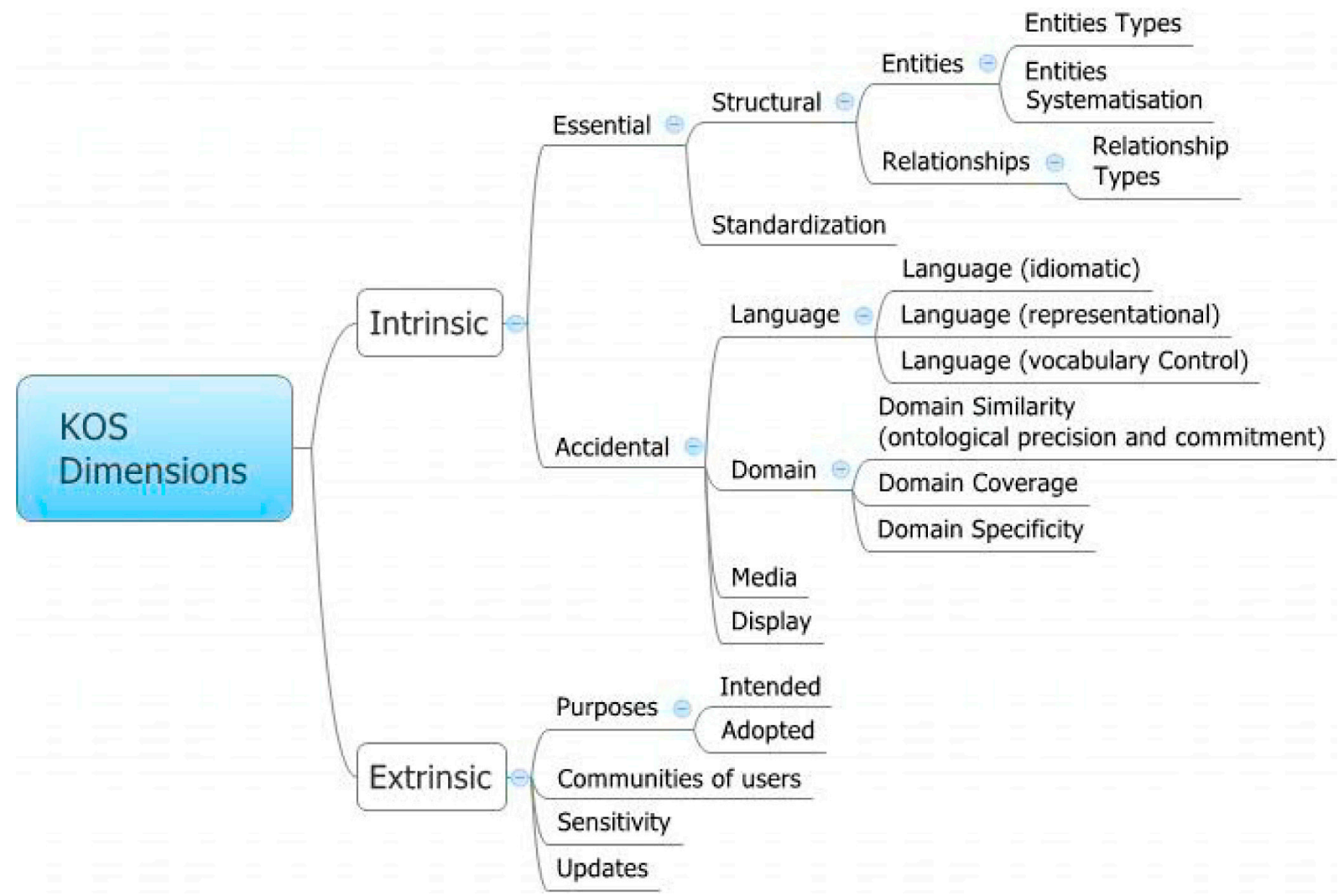

Figure 10. Proposed taxonomy of KOS dimensions

we have entities types (e.g., words, strings, numbers, concepts) and entities systematisation (e.g., random, alphabetic, systematic, enumerative). For the relationships, we have the relationship types that are present in the KOS (e.g., loose hierarchies, synonym, hyponym/hyperonym, meronomy). A KOS needs to be evaluated over the types of entities represented and the set of relationships present.

Standardization is related to the existence of a body of standards that might set rules or guidelines on the specific KOS structure (e.g., ANSI/NISO Z39.19, BS 5723:1987).

The intrinsic/accidental elements are language, domain, media, and display. Language, in its turn, is divided into idiomatic (monolingual, multilingual), representational (e.g., plain text, markup languages, diagrammatic, formal languages), and vocabulary control (e.g., natural language, controlled language, artificial language). The domain dimension is divided into similarity, coverage, and specificity. Similarity reflects the relationship of the KOS entities to the application domain (i.e., a measure of the quality of the representation, ontological precision and commitment, strongly/weakly related domain concepts). Coverage states the depth to which the domain is co- vered (e.g., shallow, deep), and specificity relates how specific the KOS is to the domain (i.e., task based, cross-domain, domain specific, foundational/upper ontology). Media is related to the informational substratum (e.g., printed, digital), and display informs the way information is presented (e.g., graphical, symbolic, plain textual, systematic textual).

The extrinsic dimensions are purposes (e.g., terminology control, indexing, classification, knowledge representation), and this is divided between Intended (planned) and Adopted, to take account of the difference between a priori and a posteriori kinds of use; communities of users (e.g., librarians, webdesigners, translators); sensitivity (e.g., classified, unclassified, public available) and updates (e.g., no update, user updatable).

We have considered how best to represent the taxonomy and future work could consider more elaborate representations if requirements warranted. For example, there are various issues of how to express the different possible relationships between KOS expressiveness (say) and the other dimensions, as well as dealing with polyhierarchies appropriately. It is also difficult to distinguish the many different kinds of "is a" relationships between concepts. Another issue is 
whether the knowledge structure should include particular instances of configurations of the typology. For example, it would be possible to employ a thesaurus that allowed polyhierarchy and specialization of the hierarchical relationships. Alternatively, a formal ontology could be used to express the full set of dimensions, instances, and classification scales.

\subsection{Conclusions}

The spectra of KOS types, mainly from Semantic Web literature, reviewed in this paper tend to make the basis for comparison a single dimension. The visual rhetoric is that of a linear progression, with ontologies as the ultimate form. This stems from the general basis of the spectra being KOS properties for logical reasoning. However, there are many potential applications for KOS and many possible dimensions for comparison. It might even be argued that more applications of KOS (including ontologies) within Semantic Web applications to date have been variations of the traditional LIS applications of browsing, search, and personalisation than applications of logical inference. Focusing on a single dimension obfuscates the underlying similarities and differences between different types and instances of KOS and hinders the selection of an appropriate KOS for a particular purpose. As a contribution to the evolving discussion, a more complex taxonomy (Figure 10) is presented for comparing and classifying KOS, as delineated in the previous section. This taxonomy allows KOS classification according to a set of reasonably independent dimensions, therefore breaking with the previous representations as continua or linear spectra.

In our view, the elements of the spectra reviewed in this paper can be considered emergent properties of those presented in the taxonomy, and can potentially be derived by taking some or many of them in account. For instance, "Complexity" or "Semantic Strength" of a KOS might be related to the structure (kinds of entities and relationships), the representational language chosen and also to the domain coverage aspects.

The taxonomy makes the distinction between ideal KOS types and particular instances, which has tended to be ignored in previous work. It does not rely on conventional labels for KOS types which tend not to have any very precise definition. Thus it can be used to categorise both KOS types and specific instances of a given type. The multi-dimensional typology of characteristics allows detailed comparison and classification. For example, hybrid forms or KOS instances that do not follow any conventional type can be described and distinguished.

Feedback after an initial presentation of the work (Souza et al. 2010) queried whether purposes might alternatively be considered a primary factor, determining KOS structure and essential characteristics. While we agree that purpose is an overlooked and important element, we wanted to allow for some element of repurposing and appropriation by user communities and hence the current placement within the taxonomy.

There are many aspects by which KOS can be classified, and many of them cannot be taken in isolation, as they are cross-dependent. In the future, an extended taxonomy might address not only the KOS as an information structure archetype, but also a specific product and its possible derived instances, displays and codifications, in a similar manner to how the FRBR treats works, expressions, manifestations, and items for bibliographic records (IFLA 2009).

There is considerable scope for future work on further defining and exploring the elements of purpose, particularly for KOS where purpose has tended to be implicit. Even within Semantic Web applications, KOS have served various different purposes and have been applied to domain objects in different ways. For example, formal ontologies and thesauri have originated from different traditions of use and communities of practice. Accordingly, there tends to be confusion about the respective purposes of each type of KOS. This is particularly manifested in the consideration of the relationship between the KOS entities and the domain objects to which they are applied. Is it some form of Instance relationship (as between an ontology class and an object in the world being modeled), or is it some form of Aboutness relationship, as is between a thesaurus or classification concept and an information resource being indexed or classified? These are very different relationships. Furthermore, an ontology would tend to encompass instance relationships and representations of objects within the body of the ontology. However, a thesaurus, for example, would not extend to a specification of the indexing language and resources being indexed. This lack of specificity when describing purpose and use complicates the process of comparing the applicability of different types of KOS. There is more work to be done on developing this analysis.

The taxonomy proposed is not the only possible configuration of the KOS elements described, although we believe the current arrangement has merits. We hope that it serves to highlight the complex 
factors involved in KOS categorisation, which cannot be captured by a one dimensional treatment and which tend to be obscured by assuming a single purpose.

\section{References}

Almeida, Maurício B., Souza, Renato, and Fonseca, Fred. 2011. Semantic in the semantic web: a critical evaluation. Knowledge organization 38: 187-203.

Bergman, Michael K. 2007. An intrepid guide to ontologies. Available http://www.mkbergman.com//? $\mathrm{p}=374$.

British Standards Institute. 2007. BS 8723-3:2007: Structured vocabularies for information retrieval: guide: vocabularies other than thesauri. Available www.standardsuk.com/shop/products_view.php? $\operatorname{prod}=44141$.

Daconta, Michael C., Obrst, Leo Joseph, and Smith, Kevin T. 2003. The semantic web: a guide to the future of $X M L$, web services, and knowledge management. Indianapolis, Ind.: Wiley.

Dextre Clarke, Stella G. 2012. ISO 25964: a standard in support of KOS interoperability. In Gilchrist, Alan, and Vernau, Judi eds. Facets of knowledge organization, proceedings of the ISKO UK biennial conference, 4th - 5th July, 2011, London. London: Emerald. Available www.iskouk.org/conf 2011/papers/dextreclarke.pd.

Guarino, Nicola. 2006. Ontology and terminology: how can formal ontology help concept modeling and terminology? In EAFT-NordTerm ws on terminology, concept modeling and ontology, Vaasa, February 10th, 2006. Available www.eaft-aet.net/ fileadmin/files/VAKKI/nicola_guarino.pdf.

Guarino, Nicola. 1998. Formal ontology and information systems. In Guarino, Nicola. ed. Formal ontology in information systems: proceedings of the first international conference (FOIS'98), June 6-8, Trento, Italy. Amsterdam: IOS Press, pp. 3-15.

Hodge, Gail. 2000. Systems of knowledge organization for digital libraries: beyond traditional authority files. Washington, D.C.: The Digital Library Federation Council on Library and Information Resources. Available www.clir.org/pubs/abstract/ pub91abst.html.

IFLA. 2009. Functional requirements for bibliographic records: final report. Available www.ifla.org/files/ cataloguing/frbr/frbr_2008.pdf.

Klein, Gunnar O., and Smith, Barry. 2005. Concept systems and ontologies. Available www.ontology. buffalo.edu/concepts/ConceptsandOntologies.pdf.
Lassila, Ora, and McGuinness, Deborah L. 2001. The role of frame-based representation on the semantic web. Knowledge Systems Laboratory Report KSL01-02. Stanford, Calif.: Stanford University. Available http://www.ksl.stanford.edu/KSL_Abstracts/ KSL-01-02.html.

McGuinness, Deborah L. 2003. Ontologies come of age. In Fensel, Dieter, Hendler, James, Lieberman, Henry, and Wahlster, Wolfgang eds. Spinning the semantic web: bringing the world wide web to its full potential. Cambridge, Mass.: MIT Press, pp. 171-96.

NISO, 2005, ANSI/NISO Z39.19-2003: guidelines for the construction, format, and management of monolingual thesauri. 2005.

Obrst, L., 2004, Ontologies E the Semantic Web for Semantic Interoperability. <www.web-services.gov/ OntologiesSemanticWebSemInteropSICOP909Obrst.ppt>.

Smith, B., and Welty, C. 2001. Ontology: towards a new synthesis. In Welty, Chris, and Smith, Barry eds. Proceedings of the international conference on Formal ontology in information systems (FOIS '01) - Volume 2001. Ongunquit, Maine: ACM Press, pp. iii-x. Available portal.acm.org/citation.cfm? doid $=505168.505201$.

Soergel D. 2001a. The representation of knowledge organization structure (KOS) data: a multiplicity of standards. Paper presented at Classification crosswalks: bringing communities together, the 4th NKOS workshop at ACM-IEEE joint conference on digital libraries (JCDL), June 28th, Roanoke, VA. Available http://nkos.slis.kent.edu/DL01 workshop.htm.

Soergel, Dagobert. 2001b. Evaluation of knowledge organization systems (KOS): characteristics for describing and evaluating KOS. Paper presented at Classification crosswalks: bringing communities together, the 4th NKOS workshop at ACM-IEEE joint conference on digital libraries (JCDL), June 28th, Roanoke, VA. Available nkos.slis.kent.edu/ 2001/SoergelCharacteristicsOfKOS.doc.

Souza, Renato Rocha, Tudhope, Douglas, and Almeida, Maurício Barcellos. 2010. The KOS spectra: a tentative faceted typology of knowledge organization systems. In Gnoli, Claudio, Mazzocchi, Fulvio eds. Paradigms and conceptual systems in knowledge organization: proceedings of the eleventh international ISKO conference 23 - 26 February 2010, Rome, Italy. Würzburg: Ergon-Verlag, pp. 122-28.

Tudhope, Douglas, Alani Harith, and Jones Christopher. 2001. Augmenting thesaurus relationships: possibilities for retrieval. Journal of digital informa- 
tion 1n8. Available http://journals.tdl.org/jodi/ article/view/181/160.

Tudhope, Douglas, Koch, Traugott, and Heery, Rachel. 2006. Terminology services and technology: JISC state of the art review. Available www.jisc.ac.uk/media/ documents/programmes/capital/terminology services_and_technology_review_sep_06.pdf.

Tudhope, Douglas. 2006. A tentative typology of KOS: towards a KOS of KOS? In NKOS Workshop, ECDL September 21, 2006 Alicante, Spain. Available www.ukoln.ac.uk/nkos/nkos2006/presentations/ tudhope.ppt.

W3C. 2009. SKOS simple knowledge organization system. Available www.w3.org/2004/02/skos/.

Welty, Chris. 1999. Ontologies: expert systems all over again? Available http://www.cs.vassar.edu/ weltyc/aaai-99/.
Wright, Sue Ellen. 2005. ISO TC 37 Standards: basic principles of terminology. In NKOS JCDL 2005 Workshop, June 7-11 2005, Denver, Colorado. Available nkos.slis.kent.edu/2005workshop/TC37.ppt.

Wright, Sue Ellen. 2008. Typology for KRRs. In NKOS-CENDI 2008. Available nkos.slis.kent. edu/2008workshop/SueEllenWright.pdf.

Zeng, Marcia Lei. 2008. Knowledge organization systems. Knowledge organization 35: 160-82.

Zeng, Marcia Lei, and Salaba, Athena. 2005. Toward an international sharing and use of subject authority data. (PowerPoint presentation) In FRBR workshop, OCLC. Available www.oclc.org/research/ events/frbr-workshop/presentations/zeng/Zeng_ Salaba.ppt. 